Preface

\title{
Coagulation and Brain
}

\author{
Benjamin Brenner, MD ${ }^{1}$ Jean-Christophe Gris, MD, PhD² \\ ${ }^{1}$ Department of Hematology, Rambam Health Care Campus, \\ Technion, Israel Institute of Technology, Haifa, Israel \\ 2 Department of Haematology, University Hospital, Nîmes and \\ University of Montpellier, GHU Caremeau, Nîmes, France \\ Semin Thromb Hemost 2013;39:847-848.
}

Blood coagulation is critical in brain homeostasis. The presence of the calvarial shield and the cerebrospinal fluid manifests the physiological need to reduce the risk for cerebral hemorrhage and its potential disastrous consequences. The brain and the placenta are the organs where tissue factor (TF) is most abundant. While this may have an impact on bleeding prevention, increased TF expression can induce disseminated intravascular coagulation following brain injury. Brain hemostasis is crucial throughout the whole life period. Although the fetus is relatively protected in the uterine milieu, hemorrhagic disease of the newborn was a major cause of disability and death until the introduction of vitamin $\mathrm{K}$ prophylaxis. However, intraventricular hemorrhage remains a detrimental problem in early deliveries. Notably, vitamin K, in addition to its role in carboxylation of coagulation factors in the liver hepatocytes, has local physiological effects on the brain as discussed in the article by Ferland. $^{1}$

Traumatic brain injury is the leading cause of death in young individuals. It is well established that the common clinical presentations of ischemic and hemorrhagic stroke are associated with hypercoagulability and hemostatic disorders. Emerging data suggest the involvement of coagulation pathways in brain physiology as well as in neurological and psychiatric disorders.

Brain vasculature is uniquely programmed to protect central nervous system (CNS) tissues and respond to their metabolic demands. The CNS can be characterized as a group of networks that interact with which each other. Neuronmicrovascular interactions at the neurovascular unit are essential for normal functioning of the CNS and maintenance of hemostasis. Responses of the hemostatic system in case of focal cerebral ischemia, the effect of anticoagulants and antithrombotics on ischemic stroke, the complex relationships between the plasminogen activator system and the CNS, and finally, the crucial role of matrix degradation in CNS focal ischemia are reviewed in the article by del Zoppo et al. ${ }^{2}$

Address for correspondence Benjamin Brenner, MD, Department of Hematology, Rambam Health Care Campus, Technion, Israel Institute of Technology, POB 9602 Haifa, Israel (e-mail: b_brenner@rambam. health.gov.il).
Thrombin and other proteases have the potential to stimulate brain inflammation through the protease activated receptors (PARs). PAR-1 is located in the synapses and its excessive activation leads to blocks in nerve conduction. These findings, according to Chapman et al, suggest that coagulation-like pathways play a significant role in the pathogenesis of inflammatory diseases in the CNS and present a target for therapeutic strategies. ${ }^{3}$

In their article, Magnus et al state that TF pathway represents a paradigm, and its impact on tumor dormancy, inflammation, angiogenesis, formation of cancer stem cell niches, and dissemination is a subject of considerable interests. ${ }^{4} \mathrm{TF}$ expression is elevated in several types of brain tumors, leading to activation of the coagulation system observed in brain tumors such as glioblastoma multiforme. Both TF expression and its vesicular emission are regulated by tumor microenvironment (e.g., hypoxia), in concert with activated oncogenic and growth factor pathways.

Polytraumatic injury results in TF release from damaged cells. The acute coagulopathy of trauma occurs early and results from significant tissue injury and tissue hypoperfusion. Head injury in the presence of polytrauma appears to increase coagulation activation and requires close monitoring and appropriate management as discussed by McCully and Schreiber. ${ }^{5}$

Chronic stressors elicit an ongoing low-grade hypercoagulable state that might impair the vasculature. Austin et al, in their article, discuss how through activation of the sympathetic nervous system, cognitive processes and corticolimbic brain areas can shape an acute prothrombotic stress response. ${ }^{6}$ The hypothalamic-pituitary adrenal axis and autonomic dysfunction are important regulators of hemostatic activity in chronic stress. Restoring normal hemostatic function with biobehavioral and pharmacological interventions bears the potential of decreasing thrombotic risk.

Lauw et al discuss cerebral vein thrombosis (CVT) as a challenge in young women on hormonal therapy or during pregnancy. ${ }^{7}$ Although there is a strong association between
Copyright @ $\odot 2013$ by Thieme Medical Publishers, Inc., 333 Seventh Avenue, New York, NY 10001, USA.

Tel: +1(212) 584-4662.
DOI http://dx.doi.org/ $10.1055 / \mathrm{s}-0033-1358662$. ISSN 0094-6176.
Brain; Guest Editors, Benjamin

Brenner, MD, and Jean-Christophe Gris, MD, PhD. 
CVT and thrombophilia, the relevance of thrombophilia testing in patients with CVT is debatable.

Recent evidence suggests an association between glucocerebrosidase (GBA) gene mutations in patients with Gaucher disease and parkinsonism (PD), which are analyzed by Rosenbaum et al. ${ }^{8}$ Hypercoagulability is reported in patients with Gaucher disease as well as in PD. Clarifying the pathophysiology of PD associated with GBA mutations may contribute to better understanding of liposomal pathway disorders and development of novel therapeutic strategies.

Gris and Brenner discuss the issue of relation between antiphospholipid antibodies (aPL) and a wide range of neurological, psychological, and psychiatric manifestations. ${ }^{9}$ In addition to purely thrombotic neurological presentations, the underlying pathophysiological mechanisms of other described neuro-psychiatric associations are not fully elucidated. The magnitude of the association and cellular and molecular mechanisms involved need to be further explored.

Some evidence suggests that tissue plasminogen activator (tPA) and plasminogen activator inhibitor-1 (PAI-1) imbalance may play an important role in pathophysiology of mental and thromboembolic disorders. The subject of coagulation involvement in psychiatric disorders is reviewed by Hoirisch-Clapauch et al. ${ }^{10}$ Patients with psychosis, or severe depression, are at increased risk for thromboembolism, with the latter being characterized by high PAI-1 levels. Estrogen inhibits synthesis of transforming growth factor- $\beta$ (TGF- $\beta$ ), while progesterone induces brain-derived neurotrophic factor release, stimulating tPA expression in neurons. Conditions related to a hypofibrinolytic status, such as polycystic ovary syndrome and metabolic syndrome, are associated with an increased risk for both depression and cardiovascular events.

Schizophrenia is a chronic, severe, and disabling mental disorder in which morphologic imaging and postmortem studies have provided evidence for localized brain atrophy. Data indicating that abnormal tPA activity is an important predisposing condition are analyzed by Hoirisch-Clapauch and Nardi. ${ }^{11}$ tPA itself, or through activation of plasminogen into plasmin, participates in synaptic regulation and plasticity, integrity of the blood-brain barrier and neurogenesis, which are crucial to memory, learning, and emotions. Low levels of tPA or loss of tPA function are reviewed as critical components of schizophrenia pathophysiology, calling for translational trials investigating the impaired PA hypothesis.

In the next article, Yamada ${ }^{12}$ discusses cerebral amyloid angiopathy (CAA)-related disorders, including hemorrhagic and ischemic stroke as well as dementia. Currently, risk factors for bleeding in patients with CAA are established. Future strategies are warranted for protection of vessel walls against amyloid-related vascular damage and development of antiamyloid therapies for CAA.
In the next article, Yamada ${ }^{12}$ discusses cerebral amyloid angiopathy (CAA)-related disorders, including hemorrhagic and ischemic stroke as well as dementia. Currently, risk factors for bleeding in patients with CAA are established. Future strategies are warranted for protection of vessel walls against amyloid-related vascular damage and development of antiamyloid therapies for CAA.

Intracerebral hemorrhage (ICH) related to oral anticoagulation (OAC) is a particularly disastrous form of stroke. Common risk factors of ICH (such as, age and hypertension), the intensity of anticoagulation, previous ischemic stroke, and the presence of cerebral vasculopathies (e.g., amyloid angiopathy, subcortical hypertensive arteriopathy) are associated with a greater risk of brain hemorrhage. Veltkamp et al discuss the hallmark of emergency treatment for vitamin K antagonist-ICH which is rapid restoration of effective coagulation using hemostatic factors. ${ }^{13}$ Management of ICH in patients on the new direct OAC is a major challenge in view of the lack of an antidote to this emerging mode of therapy. We hope that this volume will serve as a valuable source of information and will stimulate further research on coagulation and brain.

\section{References}

1 Ferland G. Vitamin K and brain function. Semin Thromb Hemost 2013;39(8):849-855

2 del Zoppo GJ, Izawa Y, Hawkins BT. Hemostasis and alterations of the central nervous system. Semin Thromb Hemost 2013;39(8): 856-875

3 Chapman J. Coagulation in inflammatory diseases of the central nervous system. Semin Thromb Hemost 2013;39(8):876-880

4 Magnus N, D’Asti E, Garnier D, Meehan B, Rak J. Brain neoplasms and coagulation. Semin Thromb Hemost 2013;39(8):881-895

5 McCully SP, Schreiber MA. Traumatic brain injury and its effect on coagulopathy. Semin Thromb Hemost 2013;39(8):896-901

6 Austin AW, Wissmann T, von Kanel R. Stress and hemostasis: an update. Semin Thromb Hemost 2013;39(8):902-912

7 Lauw MN, Barco S, Coutinho JM, Middeldorp S. Cerebral venous thrombosis and thrombophilia: a systematic review and metaanalysis. Semin Thromb Hemost 2013;39(8):913-927

8 Rosenbaum H, Aharon-Peretz J, Brenner B. Hypercoagulability, parkinsonism, and Gaucher disease. Semin Thromb Hemost 2013; 39(8):928-934

9 Gris JC, Brenner B. Antiphospholipid antibodies: neuropsychiatric presentations. Semin Thromb Hemost 2013;39(8):935-942

10 Hoirisch-Clapauch S, Nardi AE, Gris JC, Brenner B. Mental. disorders and thrombotic risk. Semin Thromb Hemost 2013;39(8): 943-949

11 Hoirisch-Clapauch S, Nardi AE. Multiple roles of tissue plasminogen activator in schizophrenia pathophysiology. Semin Thromb Hemost 2013;39(8):950-954

12 Yamada M. Brain hemorrhages in cerebral amyloid angiopathy. Semin Thromb Hemost 2013;39(8):955-962

13 Veltkamp R, Rizos T, Horstmann S. Intracerebral bleeding in patients on antithrombotic agents. Semin Thromb Hemost 2013; 39(8):963-971 